\title{
Metastatic niche functions and therapeutic opportunities
}

\author{
Toni Celià-Terrassa ${ }^{1,2}$ and Yibin Kang ${ }^{2 \star}$
}

\begin{abstract}
Metastasis is an inefficient process, especially during colonization at a distant organ. This bottleneck underlies the importance of the metastatic niche for seeding and outgrowth of metastases. Here, we classify the common functions of different metastatic niches: anchorage, survival support, protection from external insults, licensing proliferation and outgrowth. We highlight the emerging role of the metastatic niche in maintaining cancer stemness and promoting immune evasion, and discuss therapeutic opportunities against the metastatic niche.
\end{abstract}

\begin{abstract}
$\mathrm{n}$ the four decades since Raymond Schofield postulated the concept of the stem cell niche as a specialized microenvironment dictating stem cell function in hematopoiesis ${ }^{1}$, specialized cellular niches have been well characterized in different stem cell models, with demonstrated roles in balancing stem cell quiescence and activity $^{2}$. The niche is a special local site in the stromal microenvironment of stem cells that integrates signals reflecting tissue and organismal state ${ }^{1}$, and regulates stem cell fate commitment and epithelial cell plasticity during tissue homeostasis and regeneration $^{3}$. In cancer, the niche can be shaped and subverted by tumour cells ${ }^{4}$. In this context, cancer can be viewed as an ecological entity that dynamically interacts with the microenvironment and forms specialized niches to support its expansion. As in normal stem cell niches, such malignant niches also dictate tumour cell hierarchy and regulate cancer stem cell activities ${ }^{5}$. During metastasis, stemlike properties of cancer cells are required to re-initiate tumour growth in distant tissues ${ }^{6,7}$. Therefore, many parallels exist between the cancer stem cell niche and the metastatic niche at distant sites. However, the requirement of adapting to the distinct microenvironment of secondary organ sites means that the cellular composition and spatiotemporal functions of metastatic niches are often different to those of the primary tumour.
\end{abstract}

The metastatic niche model posits that disseminated tumour cells (DTCs) seek out compatible niches to support their survival and growth, and that the metastatic niche co-evolves alongside disease progression ${ }^{8,9}$. Steven Paget's 'seed and soil' hypothesis first indicated the necessity of a specific compatible 'soil' for the growth of metastases, forming the basis for the metastatic niche concept that is supported by recent experimental studies ${ }^{8-10}$. Metastatic niches can be formed either on arrival of DTCs in the recipient tissue ${ }^{9}$, or under the influence of secreted factors and/or exosomes released by the primary tumour prior to the seeding of DTCs (also termed the pre-metastatic niche $)^{10-12}$. Many studies have reported the existence of pre-metastatic niches in different organs, such as lymph nodes (LNs), lung, liver, bone and, to a lesser extent, the brain ${ }^{10}$. Metastatic niche formation also occurs through occupancy of preexisting resident stem cell niches by DTCs. This phenomenon has been observed in bone metastasis ${ }^{13}$, but remains to be elucidated in other organs.

Identifying and characterizing metastatic niches is of paramount importance for the prevention and treatment of metastatic cancer, as colonization of a distant organ is considered to be the most rate-limiting step in the metastasis cascade ${ }^{14}$. Many aspects of the spatiotemporal formation of metastatic niches remain elusive. New studies use advanced confocal and multi-photon imaging technologies, combined with genetic tools, to accurately identify metastatic niche components and functions ${ }^{15-19}$. For example, vascular endothelial growth factor receptor 3 (VEGFR3) reporter mice were developed recently to allow real-time imaging of pre-metastatic niche formation in vivo ${ }^{20}$. Moreover, sophisticated, engineered animal models have been designed to deplete specific extracellular matrix (ECM) or stromal compartments ${ }^{21}$, or to induce loss-of-function genetic changes in specific cell types, including macrophages ${ }^{22-24}$, astrocytes ${ }^{25,26}$ or endothelial cells ${ }^{27}$. These models have provided tremendous functional knowledge of the metastatic niche in vivo.

In this Review, we focus on the specialized functions of metastatic niches, with particular emphasis on their role in regulating cellular plasticity and immune interactions. Metastatic cells require cancer stem cell features and high adaptability ${ }^{6,7}$. Therefore, it is important that the niche preserves the cellular plasticity of DTCs to enable favourable immune interplay and immune editing of metastatic tumour cells. In particular, niche control of the epithelial-to-mesenchymal transition (EMT) and reverse mesenchymal-to-epithelial transition (MET) is a main modulator of cell plasticity. Throughout the Review, we focus on the dynamic relationships of the niche with the tumour cells that require intricate molecular communication, and discuss how disruption of such communication provides new therapeutic opportunities.

\section{Common functions of the metastatic niches}

The functions of the metastatic niche in supporting disease progression are multiple and can vary along the metastatic initiation process (Fig. 1). DTCs undergo physical stress in circulation and thus, following extravasation, may require immediate niche support, for instance to provide physical anchorage, survival signals, immune surveillance protection and metabolic requirements. As DTCs recover from this stress, the niche also acts to preserve and protect the cancer stem cell phenotype and cellular plasticity from differentiation cues. The elongation of this initial phase can result in metastatic dormancy, which may persist for long periods of time depending on the interplay between the niche and the metastasisinitiating-cell (MIC) abilities of the DTCs. The niche can further foster metastatic outgrowth by providing supportive growth signals and facilitating cell plasticity and immune evasion. Despite the great variety of tumour-niche interaction in different cancer types and

${ }^{1}$ Cancer Research Program, IMIM (Hospital del Mar Medical Research Institute), Barcelona, Spain. ${ }^{2}$ Department of Molecular Biology,

Princeton University, Princeton, NJ, USA. *e-mail: ykang@princeton.edu 


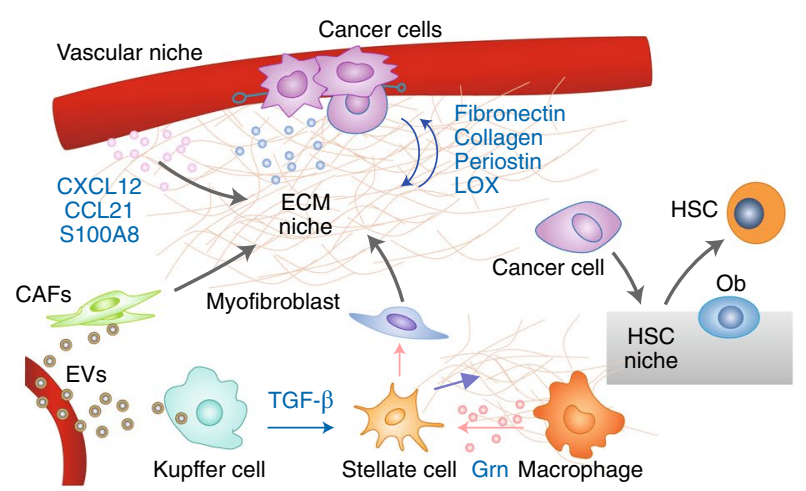

Protection

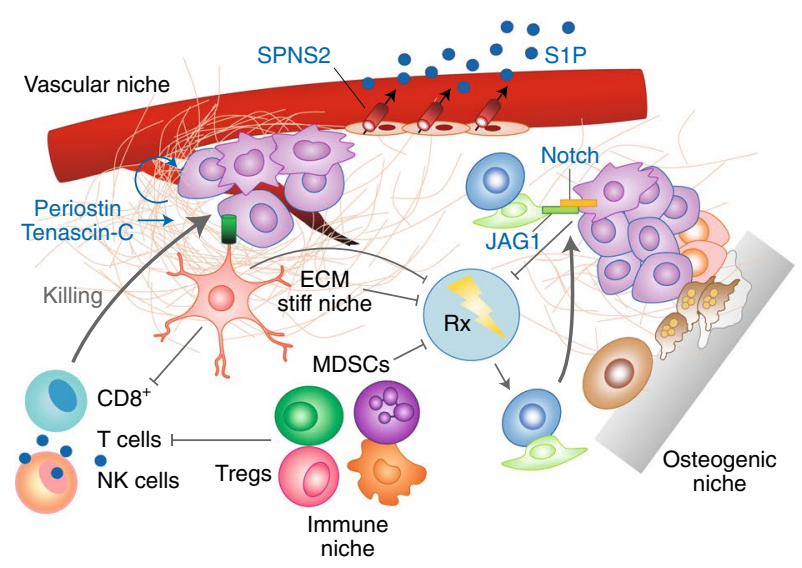

b

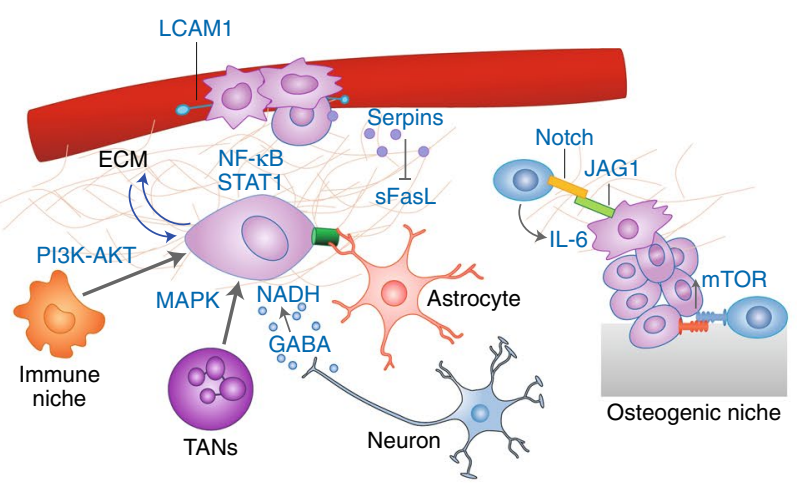

Proliferation

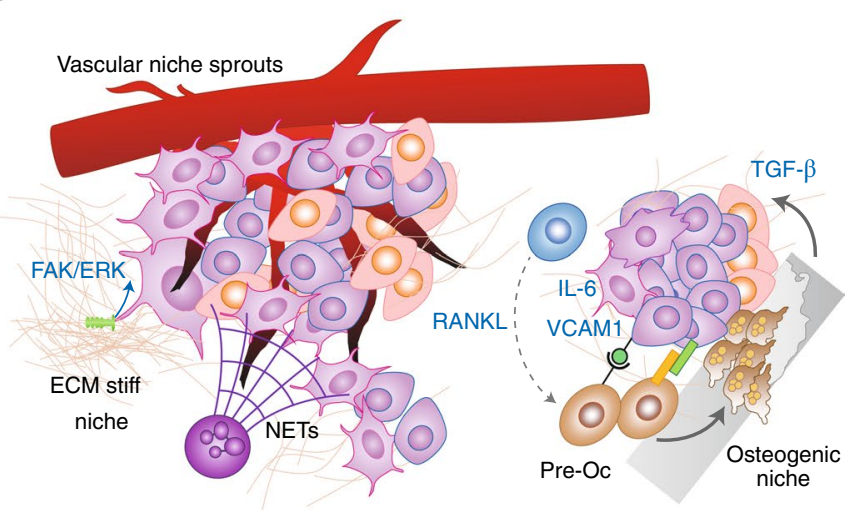

Fig. 1 | The four pillars of metastatic niche functions. a, The anchorage function is enabled by exosome- or cytokine-induced pre-metastatic niches, chemoattractants (CXCL12, CCL21 and S100A8) and a supportive substrate where cancer cells easily attach (including a vascular, ECM or HSC niche). $\mathbf{b}$, The pro-survival function is mediated by different types of niches, such as an ECM, immune, neural or osteogenic niche, that activates pro-survival signals in DTCs, such as PI3K-AKT, MAPK, NF- $\mathrm{B}$, IL-6 and mTOR. In brain metastasis, LCAM1-expressing cancer cells attach to blood vessels and secrete serpins to counteract apoptosis induced by astrocyte-derived sFasL. In addition, secreted GABA from neurons is taken up by MICs, catabolized to succinate, and converted into NADH as a source of energy for survival and growth in the brain. c, Protection against differentiation is supported by ECM components such as periostin and tenascin-C. Protection against immunosurveillance by $\mathrm{T}$ cells and NK cells is mediated by immunosuppressive immune cells (Treg cells, MDSCs, neutrophils and macrophages) and pSTAT3+-reactive astrocytes expressing PD-L1. SPNS2 is an endothelial cell transporter that pumps sphingosine-1-phosphate (S1P) from metastatic tissues into circulation, preventing $\mathrm{CD}^{+} \mathrm{T}$ cell and NK cell recruitment. This chemo-protective function is promoted by increased ECM stiffness and stroma-dependent pro-survival signals, such as myeloid-derived S100A8/9, Notch signalling, or STAT1 and NF- $\mathrm{B}$ signalling. d, Proliferative licensing of metastasis is engaged by pro-survival signals and vascular sprouting, which awakens DTCs from the dormant state. Increased ECM stiffness triggers FAK/ERK proliferative signalling. NETs, which are networks of extracellular fibres, are primarily comprised of DNA from neutrophils and facilitate the invasive expansion of metastasis. In the bone marrow, RANKL, VCAM1 and Notch signalling in pre-osteoclasts stimulate osteoclastogenesis. This initiates a cycle of bone resorption and release of growth factors that feed back to cancer cells to promote osteolytic outgrowth for bone metastasis. CAFs, cancer-associated fibroblasts; EVs, extracellular vesicles; Grn, granulin; TANs, tumour-associated neutrophils; Rx, chemotherapy; Ob, osteoblast; Pre-oc, pre-osteoclasts.

organ sites, four common functions of the niche have emerged from recent studies.

Metastatic seeding and anchorage. A key function of the premetastatic and early metastatic niche is to facilitate the landing of cancer cells, providing anchorage while circumventing anoikis and apoptosis (Fig. 1a). For example, in the perivascular niche, extravasated cancer cells remain attached to the external vessel walls, seeking physical support ${ }^{16,28}$ for recovery while remaining dormant ${ }^{29}$. Mesenchymal stem cells (MSCs), acting as pericytes surrounding the vasculature, aid this process ${ }^{30}$. The arriving DTCs further benefit from angiogenesis, lymphoangiogenesis ${ }^{20}$ and vascular leakiness induced by primary tumour-secreted extracellular vesicles ${ }^{31,32}$ or cytokines such as VEGF, which promotes endothelial FAK/Eselectin-mediated permeability ${ }^{33}$. Vascular leakiness and tumour exosomes also have pro-inflammatory effects, increasing the expression of the chemoattractant S100A8/9 (refs ${ }^{11,32}$ ), which recruit circulating tumour cells (CTCs) to the pre-metastatic lung niche ${ }^{34}$. Other chemoattractants, such as CXCL12 and CCL21, are highly expressed in common organs for metastasis (for example, LNs and lungs), and serve as homing receptors for $\mathrm{CXCR} 4^{+}$or $\mathrm{CCR}^{+}$breast cancer and melanoma cells ${ }^{35}$.

The ECM is also important in metastatic seeding, as it provides compatible substrates for tumour cell anchorage through cell surface receptors, such as integrins and CD44 (refs ${ }^{36,37}$ ). Tumourstimulated perivascular cells have been shown to enhance ECM 
production to generate a pre-metastatic niche rich in fibronectin, favouring the ensuing metastasis ${ }^{38}$. This is similar to the fibroblast-secreted fibronectin in earlier reports of pre-metastatic niches $^{12}$. Pancreatic tumour-derived exosomes stimulate transforming growth factor- $\beta$ (TGF- $\beta$ ) secretion in Kupffer liver cells, thereby inducing hepatic stellate cells to deposit fibronectin in the liver. In addition to anchoring MICs, fibronectin also favours the recruitment of bone marrow macrophages, contributing to liver pre-metastatic niche formation ${ }^{24}$. Macrophage-derived granulin activates hepatic stellate cells into myofibroblasts, which secrete periostin and favour collagen deposition to accommodate pancreatic cancer liver metastasis ${ }^{39}$. Both the composition and stiffness of the ECM are remodelled during pre-metastatic niche formation. For example, primary tumour cells secrete lysyl oxidase (LOX) into the circulation, which deposits in the lungs and cross-links collagen IV, creating a site that favours breast cancer metastasis ${ }^{40}$. Stromal cells also secrete ECM components and remodellers that promote lung metastatic colonization, such as matrix metalloproteinases (MMPs)-2 and 9 (refs ${ }^{40,41}$ ). ECM components and remodellers, such as PLOD2 and MMPs, are also produced by DTCs to generate their own ECM bed ${ }^{42,43}$, providing immediate anchorage support for lung and LN metastasis. Alternatively, in bone metastasis, normal stem cell niches can be hijacked by arriving cancer cells to provide immediate attachment and shelter. For example, prostate cancer cells out-compete hematopoietic stem cells (HSCs) and occupy the endosteal osteoblastic niche ${ }^{13}$. Interestingly, neuroblastoma cells were recently shown to disseminate through peripheral nerves to innervated $\operatorname{organs}^{44}$, suggesting that MICs may use nerves as anchorage points to initiate metastatic colonization in distant organs.

Pro-survival-signalling support. During early seeding, the signals that prevent cell death are critical (Fig. 1b). Integrin-FAK mechanotransduction through ECM engagement triggers pro-survival pathways, such as PI3K and MAPK signalling ${ }^{45}$, which are important for early survival at the metastatic site. The PI3K-AKT pathway is also activated in breast cancer cells by vascular cell adhesion molecule 1 (VCAM1)-mediated binding of recruited macrophages in lung metastasis ${ }^{46}$, and by stromal CXCL12 in the bone metastasis microenvironment ${ }^{47}$. MAPK-ERK signalling is induced in breast-MICs in the lungs by leukotrienes from recruited neutrophils ${ }^{48}$ and by S100A8/9 from recruited granulocytic-myeloid-derived suppressor cells (MDSCs) ${ }^{49}$. The osteogenic bone niche induces the pro-survival mTOR pathway in micrometastases through heterotypic cadherin signalling between osteoblasts and DTCs (ref. ${ }^{50}$ ). Tumour-derived Jagged1 (JAG1) also promotes secretion of interleukin-6 (IL-6) from osteoblasts to support bone metastatic cell survival and growth ${ }^{51}$. In the brain, $\mathrm{L} 1 C A M^{+}$metastatic tumour cells produce plasminogen activator (PA) inhibitory serpins to avoid apoptosis induced by soluble FasL from astrocytes ${ }^{16}$. On the other hand, reactive astrocytes engage pro-survival NF- $\mathrm{KB}$ signalling in MICs, fostering survival and growth ${ }^{26,52}$. Astrocyte-derived exosomes transfer miR-19a into brain MICs, which reduces PTEN levels and increases NF- $\kappa B$ signalling and CCL2 secretion to promote $\mathrm{IBA}^{+}$myeloid cell recruitment for proliferation and reduced apoptosis of MICs (ref. ${ }^{26}$ ).

The metastatic niche also satisfies the energetic demands for DTC survival. For example, tumour exosomal miR-122 suppresses glucose metabolism in pre-metastatic niche cells to increase nutrient availability for DTCs (ref. ${ }^{53}$ ). In liver metastasis, colorectal cancer cells take up creatine secreted from hepatocytes to obtain ATP and fulfil energetic requirements ${ }^{54}$. In the brain, MICs are able to use GABA released from neurons and convert it into NADH (ref. ${ }^{55}$ ).

Protection from differentiation cues, immune surveillance and therapies. A critical function of the niche is to protect tumour cells from environmental differentiation cues and immune surveillance
(Fig. 1c), supporting their stemness and survival. Additionally, the metastatic niche can thwart therapeutic interventions by increasing ECM stiffness to limit the penetration of administered drugs ${ }^{56,57}$, or by activating stroma-induced drug resistance pathways in MICs $\left(\right.$ ref. ${ }^{58}$ ). During chemotherapy, myeloid cells recruited to metastatic sites, such as the lung, can promote chemoresistance and survival of MICs by secreting S100A8/9 (ref. ${ }^{49}$ ). In brain metastasis, DTCs engage astrocyte gap-junction communication, which induces STAT1 and NF- $\mathrm{KB}$ signalling in MICs and increases their chemoresistance to carboplatin ${ }^{52}$. In bone metastasis, cisplatin or paclitaxel chemotherapy induces JAG1 expression in osteoblasts, which engages Notch signalling in breast cancer cells and enhances chemoresistance of bone metastasis ${ }^{51}$.

Fostering proliferation and outgrowth. Metastatic niches often serve as the source of growth factors that stimulate metastatic lesion outgrowth (Fig. 1d). ECM engagement of tumour cells through integrin- $\beta 1$ triggers FAK and ERK signalling, leading to rapid proliferation of micrometastases. This is further enhanced by increased ECM stiffness ${ }^{59}$ and by the formation of cell protrusions that increase ECM interactions during lung metastasis ${ }^{60,61}$. Impaired FAK/ERK signalling and soft ECM can both induce or maintain the dormant state of DTCs (ref. ${ }^{62}$ ). In the bone microenvironment, expression of VCAM1 on indolent micrometastases recruits pre-osteoclasts and promotes osteoclast differentiation to increase bone degradation and the exit of micrometastasis from dormancy towards proliferation $^{63}$. Dormancy can either be induced by metastatic niche signals or by the lack of activating signals ${ }^{62,64}$. For example, in the bone marrow, high levels of TGF- $\beta 2$ induce $p 38-\alpha / \beta$ MAPK and dormancy in DTCs (ref. ${ }^{65}$ ). In contrast, low TGF- $\beta 2$ levels in the lungs confer a more permissive niche allowing the formation of lung metasta$\operatorname{sis}^{65}$. Another mechanism of dormancy exit is through vasculature sprouting and angiogenic-switch leading to proliferation of previously dormant cells ${ }^{29}$. Despite significant progress ${ }^{64,66}$, how different organ-specific metastatic niches modulate dormancy requires further exploration.

\section{Metastatic niche regulation of plasticity and stemness}

Cellular plasticity is a core MIC characteristic that enables versatility and adaptation during metastatic colonization ${ }^{6}$, and is also critical for the epithelial homeostasis, reprogramming and tumorigenesis $^{67,68}$ underlying the phenotypic interconversion between cancer stem cells (CSCs) and non-CSCs (refs ${ }^{69,70}$ ). This plasticity is modulated by environmental cues in both normal and malignant niches, driving tumour progression and metastasis ${ }^{5,71}$. Recent studies have reported the differential needs for niche support in distant sites compared to the primary tumour site. For example, CD $44^{\text {hi }}$ $\mathrm{CD}^{+} 6^{+}$metastatic CSCs exhibit augmented lipid metabolism and consume high levels of environmental palmitic acid, promoting tumour-initiating ability at distant sites but not in the primary tumour $^{72}$. Similarly, in colorectal cancer, ablation of the $\mathrm{Lgr}^{+} \mathrm{CSC}$ population inhibited the growth of established metastases but not of primary tumors ${ }^{73}$, indicating that the distant organ microenvironment specifically supports CSCs for metastatic growth.

Different components of the niche have been reported to preserve stemness in primary tumours, which can be recapitulated in metastasis (Fig. 2). For instance, in the primary tumour niche, cancer-associated fibroblasts (CAFs) secrete multiple factors to induce stem-cell-related pathways, including Wnt and Notch signalling ${ }^{5}$. At the metastatic niche, microenvironmental TGF- $\beta$ from tumour or stromal cells induces CAFs to secrete ECM niche components, such as periostin and tenascin- $\mathrm{C}$, to maintain the stemness of metastatic cell $^{21,74}$. Periostin activates Wnt signalling ${ }^{21}$ and tenascin-C induces Notch and Lgr5 signalling ${ }^{74}$ to drive stem cell properties in breast cancer MICs. Tenascin-C is also secreted by MICs as an autonomous mechanism to preserve their stemness ${ }^{74}$. In addition, TGF- $\beta$ 


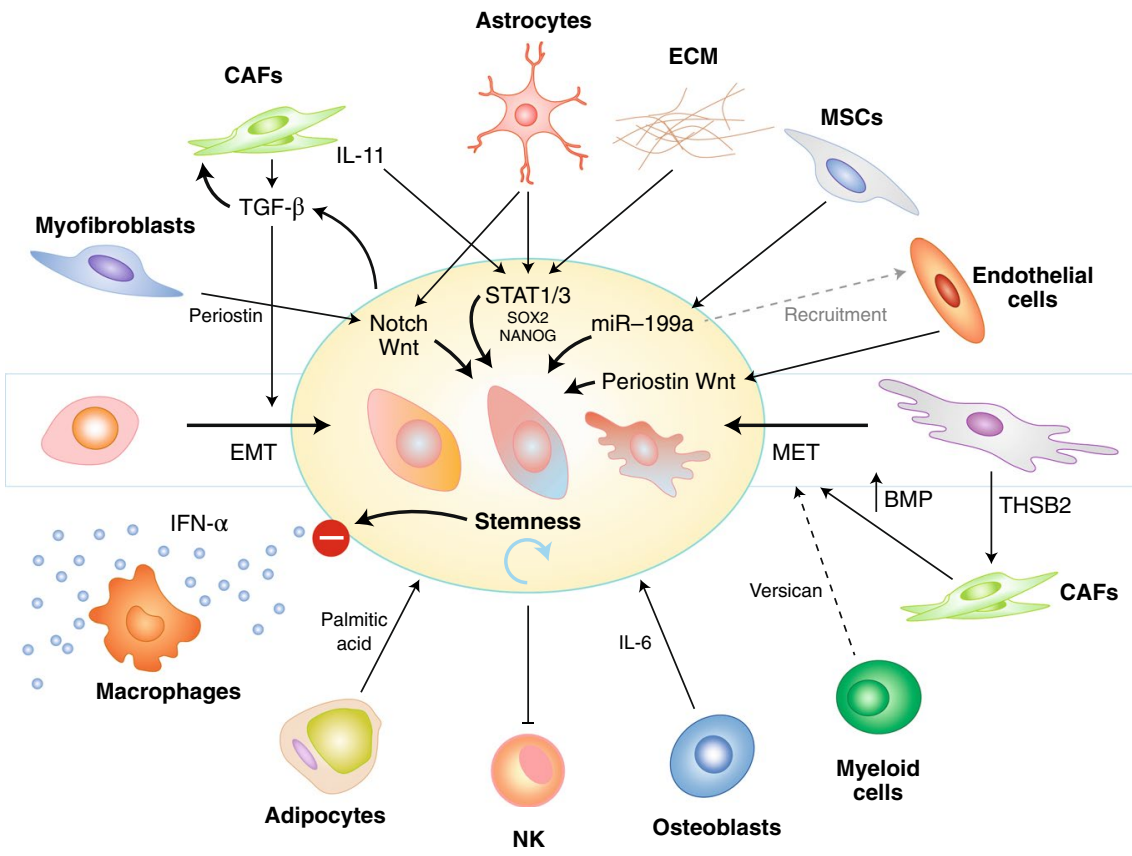

Fig. 2 | Niche regulation of stemness, EMT/MET and cell plasticity in distant sites. The metastatic niche modulates cancer cell plasticity, including the dynamic transitions between mesenchymal and epithelial states. A variety of signals from the metastatic niche dynamically orchestrate the cells to display an intermediate EMT/MET state within the 'window of stemness', as represented at the centre of the diagram, with different levels of plasticity. The main factors that the metastatic niche uses to maintain stemness are Notch, Wnt, STAT1/3, NANOG, SOX2 and IL-6, which are induced by engagement of tumour cells with different stromal cells in the niche. The stem cell-like status of MICs confers resistance to the suppressive effects of microenvironmental IFN- $\alpha$ from macrophages to evade NK cell recognition. In addition, oral carcinoma MICs are particularly dependent on the consumption of palmitic acid from adipocytes through the CD36 receptor. CAFs at distant sites, under the stimulation of thrombospondin 2 (TSHB2) produced by MICs, modulate the balance of EMT-MET transitions, inducing EMT by TGF- $\beta$ secretion and counterbalance it with bone morphogenetic protein (BMP) secretion to induce MET-thus maintaining the stemness of MICs. CAFs also reinforce stemness by secreting IL-11 to activate STAT3 in MICs. In addition, MET is induced by myeloid cells secreting versican, which activates self-renewal.

-activated fibroblasts secrete IL-11 to induce STAT3, an important transcription factor that promotes stemness of colorectal CSCs and metastasis initiation in the liver and lungs ${ }^{75,76}$.

Direct interactions between tumour cells and MSCs have been shown to induce stem cell signaling ${ }^{77}$, in part through increasing miR-199a expression in breast cancer cells ${ }^{78}$. This could also occur in the metastatic niche, as MSCs are found in multiple distant locations $^{79,80}$. miR-199a is also important for normal and breast cancer stem cell plasticity, protecting CSCs from differentiation and senescence elicited by macrophage-secreted IFN- $\alpha$ and promoting tumour initiation, both at primary and metastatic sites ${ }^{81}$. The same miRNA also promotes melanoma metastatic colonization by favouring the recruitment of endothelial cells ${ }^{82}$. Thus, miR-199a may facilitate metastasis by modulating the interaction with different components of the metastatic niche (Fig. 2). At the perivascular niche, endothelial sprouts release periostin, tenascin-C and TGF- $\beta$ in breast cancer micrometastasis to maintain stemness through Wnt and Notch signalling ${ }^{29}$. Notch signalling also promotes stemness in brain metastasis by the direct interaction of breast cancer cells with JAG1-positive astrocytes ${ }^{83}$. In lung metastasis, breast cancer cells secrete Coco to inhibit BMP signalling and prevent Wnt inhibition, which preserves the cellular plasticity needed to exit from dormancy ${ }^{84}$. Importantly, direct contact of atypical tetraspanin TM4SF1 in dormant cancer cells with collagen I at the metastatic niche mediates non-canonical DDR1-STAT3 signalling in cancer cells, leading to increased SOX2 and NANOG expression, activation of cancer stem cell properties and formation of metastasis at multiple organ $\operatorname{sites}^{85}$ (Fig. 2). On the other hand, autocrine inhibition of Wnt signalling in DTCs is important to escape immune surveillance during metastatic latency; however, these cells still display a CSC phenotype and express high levels of SOX2/SOX9, suggesting the maintenance of stemness independently of Wnt (ref. ${ }^{86}$ ). These findings underscore a critical role of the metastatic niche in regulating the stem cell properties and plasticity to favour metastatic colonization, particularly through the Wnt-Notch and STAT3-SOX2-NANOG signalling pathways (Fig. 2).

\section{Metastatic niche modulation of the EMT/MET states}

EMT is a form of cellular transdifferentiation that requires high levels of cellular plasticity, and has been linked to the stem cell gene programs $s^{67,87}$. While EMT is known to promote invasive behaviour and cancer stem cell properties, recent evidence also demonstrated the requirement of the reverse process, MET, for successful metastatic colonization in distant organ $s^{88-90}$. Indeed, extreme mesenchymal states are considered less metastatic and less plastic ${ }^{89,91,92}$, losing adaptability to hostile environments. This extreme mesenchymal differentiation is a plausible explanation for recent reports suggesting that EMT is not required for metastasis ${ }^{93,94}$. In contrast, metastatic cancer cells in partial EMT or MET states are highly plastic, with enhanced metastasis-initiation abilities ${ }^{6,61,95}$. Intermediate EMT states with metastatic ability and plasticity have been recently identified in skin and breast tumours in vivo, in a study that also showed how the primary tumour niche regulates different EMT transition states ${ }^{96}$. Thus, metastatic niches may also be important in mediating the interconversion of epithelial and mesenchymal state plasticity (Fig. 2).

Metastatic niche ECM components and stiffness can induce EMT at the metastatic site, prompting stem cell properties and 
plasticity through EMT-related signalling pathways, such as TGF- $\beta$ and IL-1 $\beta$ (refs ${ }^{97-99}$ ). In turn, EMT cells secrete ECM proteins to help construct a permissive niche for themselves ${ }^{100}$. However, in the later phase of metastasis development, TGF- $\beta$ and EMT need to be reduced to favour proliferation and metastatic outgrowth ${ }^{95,101}$. Indeed, DTCs arriving at new organs mostly display EMT-like features with stem cell gene signatures but maintain a quiescent state ${ }^{102}$. However, to progress to macrometastasis, they change to a more epithelial phenotype, downregulate stem cell gene programs related to quiescence, and express proliferative genes related to stemness, such as Myc (ref. ${ }^{102}$ ). Recent studies delve into how the metastatic niche modulates EMT reversion (Fig. 2). For example, $\mathrm{CD} 11 \mathrm{~b}^{+} \mathrm{Gr} 1^{+}$ bone marrow myeloid progenitor cells recruited in the lungs secrete versican to induce MET and proliferation in the arriving EMT-like cells $^{103}$. Similarly, pulmonary granulocytic myeloid-derived suppressor cells (MDSCs) induce MET in the arriving EMT-like cells to enhance metastatic growth ${ }^{104}$, with EMT-like cells promoting the recruitment of immature myeloid cells in the lung even before their arrival $^{105}$. Arriving EMT-like DTCs can also secrete thrombospondin-2 (THSB2) to activate lung fibroblasts, which in turn promote MET and proliferation of DTCs by activating BMP-SMAD1/5 signalling during metastatic colonization ${ }^{106}$. This MET process allows tumour cells to gain plasticity without losing stemness, supporting the notion that tumour cells in the transitional EMT/MET state are maximally plastic and potent in forming metastasis ${ }^{6,96,107}$.

\section{Tumour-immune cell interplay at metastatic sites}

Tumour cells develop strategies to modulate and recruit different immune cells with immunosuppressive effects. Bone-marrowderived myeloid cells are commonly recruited to the metastatic niche to suppress anti-metastatic immune responses in different organs (Fig. 1c). In particular, macrophages and neutrophils have been shown to suppress $\mathrm{T}$ cell infiltration and activation, thereby promoting metastasis ${ }^{108,109}$. In breast cancer metastasis, $\mathrm{Gr}^{+}$inflammatory monocytes are recruited to the lung by CCL2 released from DTCs, and then differentiate into macrophages to support metastatic seeding ${ }^{23}$. Surgical procedures can generate a systemic effect that recruits macrophages, triggering outgrowths of distant metastasis ${ }^{110}$. In fact, tumour-secreted Dockkopf-related protein 1 (DKK1) inhibits macrophage and neutrophil recruitment and thus prevents lung metastasis ${ }^{111}$. Conversely, in the bone DKK1 inhibits Wnt signalling in osteoblasts and prevents osteoprotegerinblockade of osteoclast differentiation, thereby promoting osteoclastogenesis and metastasis ${ }^{11}$. This mechanism illustrates how the same molecule may have a differential role in different organs by signalling through different cell types within the niche. Neutrophils also have important effects in the niche. For example, endothelial cells with persistently activated Notch-signalling induce VCAM1 expression, which stimulates neutrophil infiltration to facilitate melanoma lung metastasis ${ }^{112}$. Neutrophils extrude DNA-containing extracellular traps (NETs) during infectious disease ${ }^{113}$, and these NETs have been reported to increase breast cancer metastasis initiation in the lung ${ }^{114}$.

Organ-specific myeloid cells can stimulate brain metastasis growth. This is the case for astrocyte-derived exosomes, which induce recruitment of $\mathrm{IBA}^{+}$myeloid cells to promote growth of brain metastatic tumors ${ }^{26}$. In addition, brain $\mathrm{pSTAT}^{+}$reactive astrocytes express macrophage-migration inhibitory factor (MIF) to induce migration and recruitment of organ-resident $\mathrm{CD}^{+} 4^{+}$ microglia and macrophages ${ }^{25}$. Moreover, $\mathrm{pSTAT}^{+}$reactive astrocytes protect breast, lung and melanoma MICs from the immune system by forming a physical barrier and expressing programmed cell death-ligand 1 (PD-L1) to suppress infiltrating $\mathrm{CD}^{+}$ $\mathrm{T}$ cell activity ${ }^{25}$.

MDSCs, which are immature myeloid cells, are important immune-suppressors of metastasis in different organs, including the lung, liver, bone and brain ${ }^{109,115,116}$. For instance, MDSC-secreted osteopontin (OPN) reduces $\mathrm{CD}^{+} \mathrm{T}$ cell proliferation, thus exerting an immunosuppressive function at the lung metastatic niche ${ }^{117}$. Oncogenic mTOR signalling in cancer cells enhances MDSC recruitment to the lungs through granulocyte-colony stimulating factor (G-CSF), which suppresses T cell infiltration and increases metastasis initiation ${ }^{118}$. Similarly, CXCR2 signalling in neutrophil and MDSCs prevents $\mathrm{CD}^{+} \mathrm{T}$ cell infiltration and tumour cell killing in the liver metastatic niche ${ }^{119}$. Regulatory $\mathrm{T}$ cells (Tregs) have strong immunosuppressive effects on T cells and natural killer (NK) cells $^{120}$. However, their role in distant sites is less known. Tregs isolated from metastatic lymph nodes have been shown to inhibit the proliferation and activation of $\mathrm{CD} 4^{+}$and $\mathrm{CD} 8^{+} \mathrm{T}$ cells in vitro ${ }^{121}$. The balance of Tregs versus $\mathrm{CD}^{+} \mathrm{T}$ cells present in the lung parenchyma is critical for lung metastatic colonization, as elegantly illustrated in a genetic screen study using knockout mice to identify the host tissue factor Spns2 as a regulator of Treg recruitment as well as $\mathrm{NK}$ and $\mathrm{T}$ cell accumulation that modulates anti-metastatic immunity ${ }^{27}$. Together, these studies support the hypothesis that immunesuppressive cells are recruited into the metastatic niche to create a metastasis-promoting microenvironment.

\section{Metastatic niche immunoediting}

Cancer immunoediting is the process that shapes tumour immunity during primary tumour formation, and consists of three phases: elimination, equilibrium and escape ${ }^{122}$. Given that the immune context of the primary tumour site can be recapitulated in the metastatic site to a certain extent ${ }^{123}$, DTCs could potentially have been immune-edited to evade detection by the immune system even when they are still at the primary site. Nevertheless, the hostile immune environment at the metastatic site has been evidenced in multiple studies, and represents a major obstacle for metastatic colonization $^{124-128}$, suggesting that DTCs must undergo secondary immune-editing at distant organs, at least for a period of time until they are able to establish a more permissive niche. Despite the lack of direct evidence of metastatic immunoediting, in the context of adoptive immunotherapy, cycling indolent (but not quiescent) metastatic cells were immune-edited and finally escaped to form macrometastasis $^{129}$.

Although not well characterized, different degrees of secondary immunoediting likely co-evolve with the maturation of the metastatic niche. Cancer immunoediting is a long process that can last for years, particularly at the equilibrium phase ${ }^{130}$. Therefore, earlydisseminated tumour cells are likely to leave the primary tumour before completing the immune-editing process, and to require further immune editing once established at the metastatic niche ${ }^{131-133}$. Indeed, a secondary round of immune editing could explain metastatic dormancy as a prolonged equilibrium phase. This type of dormancy has long been reported as 'active suppression' by the immune system that counterbalances the proliferation rate of DTCs, thereby resulting in dynamic but steady, non-clinically detectable micrometastases ${ }^{134,135}$. Different organs have distinct resident and recruited immune cells that represent different challenges for arriving DTCs to establish metastasis ${ }^{136-138}$. Consequently, heterogeneous immune microenvironments determine different metastatic outcomes in different organs within the same individual, depending on the immune context of each organ seeded ${ }^{138}$. Therefore, immune interaction with cancer cells continues beyond the primary tumour, and immune editing likely determines the pace of metastatic colonization.

\section{Therapeutic opportunities targeting the metastatic niche}

The necessity of a metastatic niche implies the opportunity to therapeutically block its molecular and cellular interactions with DTCs to prevent or reduce metastasis. In the brain metastatic niche, astrocytes were observed to interact with DTCs through gap junctions to promote their survival ${ }^{52}$. Using the FDA approved gap junction 


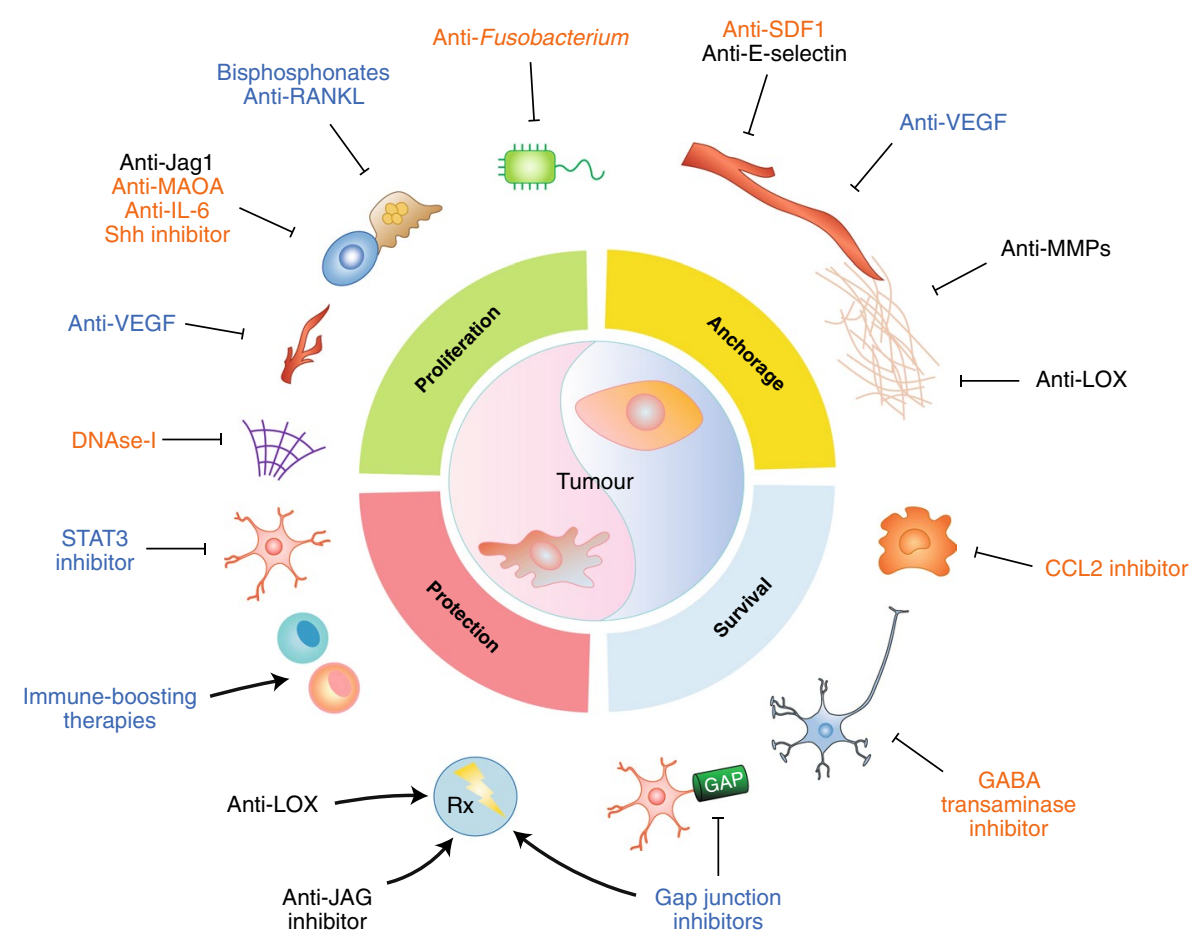

Fig. 3 | Therapeutic targeting of the metastatic niche functions. Metastatic tumour cells are dynamically changing and are regulated by four main metastatic niche functions that shelter and foster the growth of metastasis. These functions can be targeted by various therapeutic agents to block key functional molecules in the metastatic niche. Treatments with clinical evidences are represented in blue, and the others represent strategies with a potential to halt metastasis, with orange indicating FDA-approved drugs for other diseases. The anchorage function can be blocked by inhibitors of angiogenesis, ECM remodelling factors and attachment molecules, such as E-selectin. Survival of the DTCs can be compromised by targeting specific niche survival signals, such as the gap junction inhibitor, Tonabersat, that has been shown to reduce brain metastasis in clinical trials. In addition, the chemo-protective function of the niche can be disrupted by targeting specific tumour-stromal interactions, such as osteoblastic JAG1 in bone metastasis. Niche-mediated evasion from immunosurveillance can be abrogated with different types of immunotherapies, and a better understanding of the evolution of immune-editing at the metastasis niche will help optimize treatment. The pSTAT3 inhibitor Silibinin (Legasil) prevents astrocyte activation, thereby reversing the suppression of $C D 8^{+} \mathrm{T}$ cell killing activity. Finally, the proliferative license can be disrupted by controlling dormancy, blocking vascular sprouting and breaking vicious cycles in the bone metastasis (for example, using anti-JAG1, anti-RANKL and anti-IL-6 antibodies, as well as Shh inhibitors, MAOA inhibitors and bisphosphonates). FDA-approved drugs for infectious diseases also offer a great opportunity to block proliferation, with examples such as, DNase-I to clear NETs, and antibiotics (such as metronidazole) to eliminate Fusobacterium xeno-niches.

inhibitors tonabersat and meclofenamate to target brain metastasis in a pre-clinical setting showed promising results, and has been followed by clinical trials using the same strategy to treat brain metastasis (ClinicalTrials.gov; NCT02429570) ${ }^{52}$. In a separate study, use of nutraceutical Legasil (Silibinin) to inhibit STAT3 activity successfully reduced the immunosuppressive effect of reactive astrocytes and suppressed brain metastasis ${ }^{25}$. Osteoclast-targeting agents, bisphosphonates and denosumab (RANKL blocking antibody) have been clinically approved for the treatment of bone metasta$\operatorname{sis}^{139}$. A therapeutic antibody against JAG1 has been shown to block tumour-stromal interactions and displayed a strong synergy with chemotherapy in reducing bone metastasis and chemoresistance ${ }^{51}$. A monoamine oxidase A inhibitor has also been used to block Shhsecretion from cancer cells, thus preventing osteoblast stimulation and release of RANKL and IL-6 in prostate cancer metastasis ${ }^{140}$. These studies demonstrate that targeting the survival and chemoprotective function of the niche represents promising strategies for therapeutic invention of metastasis (Fig. 3).

A major problem in the clinical management of cancer is metastatic dormancy, as it presents a risk of relapse and undermines the therapeutic benefit of treatment. In addition to maintaining residual cancer cells at a dormant state, two alternative strategies have been proposed to target dormancy ${ }^{66}$. The first is to disrupt the anchorage function of the niche to enhance mobilization and vulnerability of tumour cells. For example, blockade of SDF-1 and E-selectin dislodges dormant breast cancer cells from perisinusoidal vascular regions and mobilizes dormant micrometastasis out of the bone marrow to prevent metastatic colonization ${ }^{28}$. The anchorage function of the ECM and blood vessels could also be targeted with antiLOX (ref. ${ }^{141}$ ), anti-MMPs (ref. ${ }^{142}$ ) and anti-VEGF (ref. ${ }^{143}$ )(Fig. 3). Alternatively, blocking niche-protection and survival functions, such as niche-derived factors that induce chemoresistance to DTCs, represents another dormancy-targeting regimen. Taken together, these strategies will be critical in eliminating any minimal residual disease and work towards a complete cure for the patient.

The pro-metastatic immune cells forming the metastatic niche also represent an excellent target for therapeutic intervention (Fig. 3). CCL2/CCR2-mediated macrophage recruitment in lung metastasis $^{23}$ could be blocked with CCL2 inhibitors that have been shown to have therapeutic effects in primary tumours ${ }^{144}$. Endothelial Notch1 and VCAM1-blockade prevents ovarian lung metastasis by preventing neutrophil infiltration ${ }^{112}$. In pre-clinical models, DNase-I-coated nanoparticles have been shown to be a potential therapeutic inhibitor of metastasis through digestion of DNA in NETs (ref. ${ }^{114}$ ). The inhibition of CXCR2 on MDSCs allows T cell infiltration in pancreatic liver metastasis, which enhances the efficiency of anti-PD1 immunotherapy ${ }^{119}$. Overall, immunotherapy approaches have achieved outstanding results in certain metastatic cancer types and hold great promise in oncology ${ }^{145}$. However, recent long-term follow-up studies have shown acquired resistance to the initial treatment in a quarter of melanoma 
responsive patients ${ }^{146}$. Therefore, it is critical to study the immune metastatic niche formation and its dynamic immune-editing to understand the acquired immunoresistance of MICs.

Identification of new niche functions and different types of metastatic niches will continue to increase the range of therapeutic options. The emergence of neural niches may allow use of a multitude of available neurotransmitter modulators, such as vigabatrin, to block GABA transaminase (ABAT) and to avoid GABA catabolism, which has been experimentally shown to be important in brain metastasis ${ }^{55}$. Another putative metastatic niche is the bacterial niche, which could also be classified as 'xeno-niche' as it is formed by different species. For example, Fusobacterium and its associated microbiome are found in primary and metastatic locations, and support cancer cell proliferation and growth by unknown mechanisms ${ }^{147}$. Xeno-niches represent an ideal therapeutic target by using antibiotics to directly kill the specific bacteria and abrogate their supportive function, as shown in a recent study using metronidazole ${ }^{147}$. These emerging niches will offer more opportunities to specifically target metastasis depending on location, function and timing.

\section{Concluding remarks}

In this Review, we describe the metastatic niche functions that provide a permissive environment for eventual outgrowth of DTCs in distant organ sites. We classify these functions into four main categories: anchorage, survival, protection and proliferation (Figs. 1, 3). Furthermore, two central themes are shared when considering the interaction between MICs and metastatic niches: the maintenance of cancer stemness and plasticity, and the metastasis-favouring immune interplay and editing. This is not meant to be an exhaustive list of niche functions, as on-going and future research will undoubtedly reveal further complexities. Furthermore, different organs may have different types of niches that facilitate the metastatic process, as evidenced by the organotropism of different cancer types ${ }^{139}$. Proposing common functions among different niches and in different organs is intended to provide a conceptual framework that may prove useful when considering therapeutic strategies to target metastasis. It is also important to consider that metastatic niches are constantly evolving, and thus some functions are more important at early stages and others at later stages. Therefore, future pre-clinical studies should focus on experimentally testing the critical therapeutic windows to shut down common niche functions, according to the timeline of metastatic colonization steps. For these studies, the application of advanced imaging and single cell -omics techniques will help decipher the spatiotemporal dynamics of tumour-niche interactions. Development of niche reporters or niche-specific imaging probes will be very informative regarding the dynamics of metastatic niches, especially when utilizing clinically applicable technologies such as positron emission tomography (PET) and nuclear magnetic resonance (NMR). Engineering threedimensional model systems in vitro and using niche-mimicking biomaterials that regulate functions following implantation in vivo $^{148}$, will help to deconstruct the metastatic niche. Combinatorial gene function analysis within tissues at a temporal and cellular resolution can reveal the roles of multiple niche stromal populations, as exemplified by the recently reported dual-IGF mosaic mice ${ }^{149}$. It is worth noting that many metastasis studies use experimental metastasis models in which tumours cells are introduced directly into circulation. In such models, the dynamics of metastatic niche development might be substantially different from models of spontaneous metastasis, which often take much longer to develop. Careful comparison of the metastasis niches in these different models might help provide important insights in clinical settings.

Metastasis research has been limited by the difficulty of obtaining clinical samples. This is even more challenging when studying the pre-metastatic niche, as pre-metastatic tissues are difficult to detect and not normally resected. However, experimental models and advanced technologies clearly show the importance of the niche in metastatic disease. We expect that future research into metastatic niche functions will continue to provide conceptual frameworks to develop new therapies for containing or eliminating metastatic disease. In particular, a better understanding of how the niche regulates cellular plasticity and immune evasion holds great potential for therapeutic intervention of metastasis, as both functions are required across all metastatic stages and organs.

Received: 5 February 2018; Accepted: 19 June 2018;

Published online: 26 July 2018

\section{References}

1. Schofield, R. The relationship between the spleen colony-forming cell and the haemopoietic stem cell. Blood Cells 4, 7-25 (1978).

2. Scadden, D. T. Nice neighborhood: emerging concepts of the stem cell niche. Cell 157, 41-50 (2014).

3. Blanpain, C. \& Fuchs, E. Stem cell plasticity. Plasticity of epithelial stem cells in tissue regeneration. Science 344, 1242281 (2014).

4. Barcellos-Hoff, M. H., Lyden, D. \& Wang, T. C. The evolution of the cancer niche during multistage carcinogenesis. Nat. Rev. Cancer 13, 511-518 (2013).

5. Plaks, V., Kong, N. \& Werb, Z. The cancer stem cell niche: how essential is the niche in regulating stemness of tumor cells? Cell Stem Cell 16, 225-238 (2015).

6. Celia-Terrassa, T. \& Kang, Y. Distinctive properties of metastasis-initiating cells. Genes Dev. 30, 892-908 (2016).

7. Oskarsson, T., Batlle, E. \& Massague, J. Metastatic stem cells: sources, niches, and vital pathways. Cell Stem Cell 14, 306-321 (2014).

8. Psaila, B. \& Lyden, D. The metastatic niche: adapting the foreign soil. Nat. Rev. Cancer 9, 285-293 (2009).

9. Sleeman, J. P. The metastatic niche and stromal progression. Cancer Metast. Rev. 31, 429-440 (2012).

10. Peinado, H. et al. Pre-metastatic niches: organ-specific homes for metastases. Nat. Rev. Cancer 17, 302-317 (2017).

11. Hoshino, A. et al. Tumour exosome integrins determine organotropic metastasis. Nature 527, 329-335 (2015).

12. Kaplan, R. N. et al. VEGFR1-positive haematopoietic bone marrow progenitors initiate the pre-metastatic niche. Nature 438, 820-827 (2005).

13. Shiozawa, Y. et al. Human prostate cancer metastases target the hematopoietic stem cell niche to establish footholds in mouse bone marrow. J. Clin. Invest. 121, 1298-1312 (2011).

14. Vanharanta, S. \& Massague, J. Origins of metastatic traits. Cancer Cell 24, 410-421 (2013).

15. Headley, M. B. et al. Visualization of immediate immune responses to pioneer metastatic cells in the lung. Nature 531, 513-517 (2016).

16. Valiente, M. et al. Serpins promote cancer cell survival and vascular co-option in brain metastasis. Cell 156, 1002-1016 (2014).

17. Alexander, S., Weigelin, B., Winkler, F. \& Friedl, P. Preclinical intravital microscopy of the tumour-stroma interface: invasion, metastasis, and therapy response. Curr. Opin. Cell Biol. 25, 659-671 (2013).

18. Mayorca-Guiliani, A. E. et al. ISDoT: in situ decellularization of tissues for high-resolution imaging and proteomic analysis of native extracellular matrix. Nat. Med. 23, 890-898 (2017).

19. Kienast, Y. et al. Real-time imaging reveals the single steps of brain metastasis formation. Nat. Med. 16, 116-122 (2010).

20. Olmeda, D. et al. Whole-body imaging of lymphovascular niches identifies pre-metastatic roles of midkine. Nature 546, 676-680 (2017).

21. Malanchi, I. et al. Interactions between cancer stem cells and their niche govern metastatic colonization. Nature 481, 85-89 (2012).

22. Qian, B. et al. A distinct macrophage population mediates metastatic breast cancer cell extravasation, establishment and growth. PLOS ONE 4, e6562 (2009).

23. Qian, B. Z. et al. CCL2 recruits inflammatory monocytes to facilitate breasttumour metastasis. Nature 475, 222-225 (2011).

24. Costa-Silva, B. et al. Pancreatic cancer exosomes initiate pre-metastatic niche formation in the liver. Nat. Cell Biol. 17, 816-826 (2015).

25. Priego, N. et al. STAT3 labels a subpopulation of reactive astrocytes required for brain metastasis. Nat. Med. https://doi.org/10.1038/s41591-018 0044-4 (2018).

26. Zhang, L. et al. Microenvironment-induced PTEN loss by exosomal microRNA primes brain metastasis outgrowth. Nature 527, 100-104 (2015).

27. van der Weyden, L. et al. Genome-wide in vivo screen identifies novel host regulators of metastatic colonization. Nature 541, 233-236 (2017). 
28. Price, T. T. et al. Dormant breast cancer micrometastases reside in specific bone marrow niches that regulate their transit to and from bone. Sci. Transl Med. 8, 340ra373 (2016).

29. Ghajar, C. M. et al. The perivascular niche regulates breast tumour dormancy. Nat. Cell Biol. 15, 807-817 (2013).

30. Correa, D., Somoza, R. A., Lin, P., Schiemann, W. P. \& Caplan, A. I. Mesenchymal stem cells regulate melanoma cancer cells extravasation to bone and liver at their perivascular niche. Int. J. Cancer 138, 417-427 (2016).

31. Grange, C. et al. Microvesicles released from human renal cancer stem cells stimulate angiogenesis and formation of lung premetastatic niche. Cancer Res. 71, 5346-5356 (2011).

32. Peinado, $\mathrm{H}$. et al. Melanoma exosomes educate bone marrow progenitor cells toward a pro-metastatic phenotype through MET. Nat. Med. 18, 883-891 (2012).

33. Hiratsuka, S. et al. Endothelial focal adhesion kinase mediates cancer cell homing to discrete regions of the lungs via E-selectin up-regulation. Proc. Natl Acad. Sci. USA 108, 3725-3730 (2011).

34. Hiratsuka, S. et al. The S100A8-serum amyloid A3-TLR4 paracrine cascade establishes a pre-metastatic phase. Nat. Cell Biol. 10, 1349-1355 (2008).

35. Muller, A. et al. Involvement of chemokine receptors in breast cancer metastasis. Nature 410, 50-56 (2001)

36. Seguin, L., Desgrosellier, J. S., Weis, S. M. \& Cheresh, D. A. Integrins and cancer: regulators of cancer stemness, metastasis, and drug resistance. Trends Cell Biol. 25, 234-240 (2015).

37. Yu, Q., Toole, B. P. \& Stamenkovic, I. Induction of apoptosis of metastatic mammary carcinoma cells in vivo by disruption of tumor cell surface CD44 function. J. Exp. Med. 186, 1985-1996 (1997).

38. Murgai, M. et al. KLF4-dependent perivascular cell plasticity mediates pre-metastatic niche formation and metastasis. Nat. Med. 23 1176-1190 (2017).

39. Nielsen, S. R. et al. Macrophage-secreted granulin supports pancreatic cancer metastasis by inducing liver fibrosis. Nat. Cell Biol. 18, 549-560 (2016).

40. Erler, J. T. et al. Hypoxia-induced lysyl oxidase is a critical mediator of bone marrow cell recruitment to form the premetastatic niche. Cancer Cell 15, 35-44 (2009)

41. Hiratsuka, S. et al. MMP9 induction by vascular endothelial growth factor receptor-1 is involved in lung-specific metastasis. Cancer Cell 2, 289-300 (2002).

42. Descot, A. \& Oskarsson, T. The molecular composition of the metastatic niche. Exp. Cell Res. 319, 1679-1686 (2013).

43. Gilkes, D. M. et al. Procollagen lysyl hydroxylase 2 is essential for hypoxia-induced breast cancer metastasis. Mol. Cancer Res. 11, 456-466 (2013).

44. Delloye-Bourgeois, C. et al. Microenvironment-driven shift of cohesion/ detachment balance within tumors induces a switch toward metastasis in neuroblastoma. Cancer Cell 32, 427-443 (2017)

45. Chiarugi, P. \& Giannoni, E. Anoikis: a necessary death program for anchorage-dependent cells. Biochem. Pharmacol. 76, 1352-1364 (2008).

46. Chen, Q., Zhang, X. H. \& Massague, J. Macrophage binding to receptor VCAM-1 transmits survival signals in breast cancer cells that invade the lungs. Cancer Cell 20, 538-549 (2011).

47. Zhang, X. H. et al. Latent bone metastasis in breast cancer tied to Src-dependent survival signals. Cancer Cell 16, 67-78 (2009).

48. Wculek, S. K. \& Malanchi, I. Neutrophils support lung colonization of metastasis-initiating breast cancer cells. Nature 528, 413-417 (2015).

49. Acharyya, S. et al. A CXCL1 paracrine network links cancer chemoresistance and metastasis. Cell 150, 165-178 (2012).

50. Wang, H. et al. The osteogenic niche promotes early-stage bone colonization of disseminated breast cancer cells. Cancer Cell 27, 193-210 (2015).

51. Zheng, H. et al. therapeutic antibody targeting tumor- and osteoblastic niche-derived jagged 1 sensitizes bone metastasis to chemotherapy. Cancer Cell 32, 731-747 (2017).

52. Chen, Q. et al. Carcinoma-astrocyte gap junctions promote brain metastasis by cGAMP transfer. Nature 533, 493-498 (2016).

53. Fong, M. Y. et al. Breast-cancer-secreted miR-122 reprograms glucose metabolism in premetastatic niche to promote metastasis. Nat. Cell Biol. 17, 183-194 (2015).

54. Loo, J. M. et al. Extracellular metabolic energetics can promote cancer progression. Cell 160, 393-406 (2015).

55. Neman, J. et al. Human breast cancer metastases to the brain display GABAergic properties in the neural niche. Proc. Natl Acad. Sci. USA 111, 984-989 (2014).

56. Egeblad, M., Rasch, M. G. \& Weaver, V. M. Dynamic interplay between the collagen scaffold and tumor evolution. Curr. Opin. Cell Biol. 22 697-706 (2010).

57. Vennin, C. et al. Transient tissue priming via ROCK inhibition uncouples pancreatic cancer progression, sensitivity to chemotherapy, and metastasis. Sci. Transl Med. 9, eaai8504 (2017).
58. Wan, L., Pantel, K. \& Kang, Y. Tumor metastasis: moving new biological insights into the clinic. Nat. Med. 19, 1450-1464 (2013).

59. Schrader, J. et al. Matrix stiffness modulates proliferation, chemotherapeutic response, and dormancy in hepatocellular carcinoma cells. Hepatology 53, 1192-1205 (2011)

60. Shibue, T., Brooks, M. W., Inan, M. F., Reinhardt, F. \& Weinberg, R. A. The outgrowth of micrometastases is enabled by the formation of filopodium-like protrusions. Cancer Discov. 2, 706-721 (2012).

61. Lambert, A. W., Pattabiraman, D. R. \& Weinberg, R. A. Emerging biological principles of metastasis. Cell 168, 670-691 (2017).

62. Giancotti, F. G. Mechanisms governing metastatic dormancy and reactivation. Cell 155, 750-764 (2013).

63. Lu, X. et al. VCAM-1 promotes osteolytic expansion of indolent bone micrometastasis of breast cancer by engaging alpha4beta1-positive osteoclast progenitors. Cancer Cell 20, 701-714 (2011).

64. Sosa, M. S., Bragado, P. \& Aguirre-Ghiso, J. A. Mechanisms of disseminated cancer cell dormancy: an awakening field. Nat. Rev. Cancer 14, 611-622 (2014).

65. Bragado, P. et al. TGF- $\beta 2$ dictates disseminated tumour cell fate in target organs through TGF- $\beta$-RIII and $\mathrm{p} 38 \alpha / \beta$ signalling. Nat. Cell Biol. 15, 1351-1361 (2013).

66. Ghajar, C. M. Metastasis prevention by targeting the dormant niche. Nat. Rev. Cancer 15, 238-247 (2015).

67. Varga, J. \& Greten, F. R. Cell plasticity in epithelial homeostasis and tumorigenesis. Nat. Cell Biol. 19, 1133-1141 (2017).

68. Mosteiro, L. et al. Tissue damage and senescence provide critical signals for cellular reprogramming in vivo. Science 354, aaf4445 (2016).

69. Batlle, E. \& Clevers, H. Cancer stem cells revisited. Nat. Med. 23 1124-1134 (2017).

70. Gupta, P. B. et al. Stochastic state transitions give rise to phenotypic equilibrium in populations of cancer cells. Cell 146, 633-644 (2011).

71. Hendrix, M. J. et al. Reprogramming metastatic tumour cells with embryonic microenvironments. Nat. Rev. Cancer 7, 246-255 (2007)

72. Pascual, G. et al. Targeting metastasis-initiating cells through the fatty acid receptor CD36. Nature 541, 41-45 (2017).

73. de Sousa e Melo, F. et al. A distinct role for Lgr5+ stem cells in primary and metastatic colon cancer. Nature 543, 676-680 (2017).

74. Oskarsson, $\mathrm{T}$. et al. Breast cancer cells produce tenascin $\mathrm{C}$ as a metastatic niche component to colonize the lungs. Nat. Med. 17, 867-874 (2011).

75. Yu, H., Lee, H., Herrmann, A., Buettner, R. \& Jove, R. Revisiting STAT3 signalling in cancer: new and unexpected biological functions. Nat. Rev. Cancer 14, 736-746 (2014).

76. Calon, A. et al. Dependency of colorectal cancer on a TGF-beta-driven program in stromal cells for metastasis initiation. Cancer Cell 22, 571-584 (2012)

77. Roorda, B. D., Elst, A., Boer, T. G., Kamps, W. A. \& de Bont, E. S. Mesenchymal stem cells contribute to tumor cell proliferation by direct cell-cell contact interactions. Cancer Invest. 28, 526-534 (2010).

78. Cuiffo, B. G. et al. MSC-regulated microRNAs converge on the transcription factor FOXP2 and promote breast cancer metastasis. Cell Stem Cell 15, 762-774 (2014).

79. Ren, G., Esposito, M. \& Kang, Y. Bone metastasis and the metastatic niche. J. Mol. Med. 93, 1203-1212 (2015).

80. Ridge, S. M., Sullivan, F. J. \& Glynn, S. A. Mesenchymal stem cells: key players in cancer progression. Mol. Cancer 16, 31 (2017).

81. Celia-Terrassa, T. et al. Normal and cancerous mammary stem cells evade interferon-induced constraint through the miR-199a-LCOR axis. Nat. Cell Biol. 19, 711-723 (2017).

82. Pencheva, N. et al. Convergent multi-miRNA targeting of ApoE drives LRP1/LRP8-dependent melanoma metastasis and angiogenesis. Cell 151, 1068-1082 (2012).

83. Xing, F. et al. Reactive astrocytes promote the metastatic growth of breast cancer stem-like cells by activating Notch signalling in brain. EMBO Mol. Med. 5, 384-396 (2013).

84. Gao, H. et al. The BMP inhibitor Coco reactivates breast cancer cells at lung metastatic sites. Cell 150, 764-779 (2012).

85. Gao, H. et al. Multi-organ site metastatic reactivation mediated by non-canonical discoidin domain receptor 1 signaling. Cell 166, 47-62 (2016).

86. Malladi, S. et al. Metastatic latency and immune evasion through autocrine inhibition of WNT. Cell 165, 45-60 (2016).

87. Mani, S. A. et al. The epithelial-mesenchymal transition generates cells with properties of stem cells. Cell 133, 704-715 (2008).

88. Korpal, M. et al. Direct targeting of Sec23a by miR-200s influences cancer cell secretome and promotes metastatic colonization. Nat. Med. 17, 1101-1108 (2011).

89. Ocana, O. H. et al. Metastatic colonization requires the repression of the epithelial-mesenchymal transition inducer Prrx1. Cancer Cell 22, 709-724 (2012). 
90. Tsai, J. H., Donaher, J. L., Murphy, D. A., Chau, S. \& Yang, J. Spatiotemporal regulation of epithelial-mesenchymal transition is essential for squamous cell carcinoma metastasis. Cancer Cell 22, 725-736 (2012).

91. Celia-Terrassa, T. et al. Epithelial-mesenchymal transition can suppress major attributes of human epithelial tumor-initiating cells. J. Clin. Invest. 122, 1849-1868 (2012)

92. Tran, H. D. et al. Transient SNAIL1 expression is necessary for metastatic competence in breast cancer. Cancer Res. 74, 6330-6340 (2014).

93. Fischer, K. R. et al. Epithelial-to-mesenchymal transition is not required for lung metastasis but contributes to chemoresistance. Nature 527, 472-476 (2015).

94. Zheng, X. et al. Epithelial-to-mesenchymal transition is dispensable for metastasis but induces chemoresistance in pancreatic cancer. Nature 527, 525-530 (2015).

95. Nieto, M. A., Huang, R. Y., Jackson, R. A. \& Thiery, J. P. Emt: 2016. Cell 166, 21-45 (2016)

96. Pastushenko, I. et al. Identification of the tumour transition states occurring during EMT. Nature 556, 463-468 (2018).

97. Kalluri, R. \& Weinberg, R. A. The basics of epithelial-mesenchymal transition. J. Clin. Invest. 119, 1420-1428 (2009).

98. Lopez-Novoa, J. M. \& Nieto, M. A. Inflammation and EMT: an alliance towards organ fibrosis and cancer progression. EMBO Mol. Med. 1, 303-314 (2009).

99. Wei, S. C. et al. Matrix stiffness drives epithelial-mesenchymal transition and tumour metastasis through a TWIST1-G3BP2 mechanotransduction pathway. Nat. Cell Biol. 17, 678-688 (2015)

100. Thiery, J. P., Acloque, H., Huang, R. Y. \& Nieto, M. A. Epithelialmesenchymal transitions in development and disease. Cell 139, 871-890 (2009).

101. Massague, J. TGF- $\beta$ in cancer. Cell 134, 215-230 (2008)

102. Lawson, D. A. et al. Single-cell analysis reveals a stem-cell program in human metastatic breast cancer cells. Nature 526, 131-135 (2015).

103. Gao, D. et al. Myeloid progenitor cells in the premetastatic lung promote metastases by inducing mesenchymal to epithelial transition. Cancer Res. 72, 1384-1394 (2012).

104. Ouzounova, M. et al. Monocytic and granulocytic myeloid derived suppressor cells differentially regulate spatiotemporal tumour plasticity during metastatic cascade. Nat. Commun. 8, 14979 (2017).

105. Salvador, F. et al. Lysyl oxidase-like protein LOXL2 promotes lung metastasis of breast cancer. Cancer Res. 77, 5846-5859 (2017).

106. Del Pozo Martin, Y. et al. Mesenchymal cancer cell-stroma crosstalk promotes niche activation, epithelial reversion, and metastatic colonization. Cell Rep. 13, 2456-2469 (2015).

107. Beerling, E. et al. Plasticity between epithelial and mesenchymal states unlinks EMT from metastasis-enhancing stem cell capacity. Cell Rep. 14, 2281-2288 (2016).

108. Kitamura, T., Qian, B. Z. \& Pollard, J. W. Immune cell promotion of metastasis. Nat. Rev. Immunol. 15, 73-86 (2015).

109. Condamine, T., Ramachandran, I., Youn, J. I. \& Gabrilovich, D. I. Regulation of tumor metastasis by myeloid-derived suppressor cells. Annu. Rev. Med. 66, 97-110 (2015).

110. Krall, J. A. et al. The systemic response to surgery triggers the outgrowth of distant immune-controlled tumors in mouse models of dormancy. Sci. Transl Med. 10, eean3464 (2018).

111. Zhuang, X. et al. Differential effects on lung and bone metastasis of breast cancer by Wnt signalling inhibitor DKK1. Nat. Cell Biol. 19, 1274-1285 (2017).

112. Wieland, E. et al. Endothelial Notch 1 activity facilitates metastasis. Cancer Cell 31, 355-367 (2017).

113. Branzk, N. \& Papayannopoulos, V. Molecular mechanisms regulating NETosis in infection and disease. Semin. Immunopathol. 35, 513-530 (2013).

114. Park, J. et al. Cancer cells induce metastasis-supporting neutrophil extracellular DNA traps. Sci. Transl Med. 8, 361 ra138 (2016).

115. Marvel, D. \& Gabrilovich, D. I. Myeloid-derived suppressor cells in the tumor microenvironment: expect the unexpected. J. Clin. Invest. 125, 3356-3364 (2015).

116. Liu, Y. et al. Premetastatic soil and prevention of breast cancer brain metastasis. Neuro. Oncol. 15, 891-903 (2013).

117. Sangaletti, S. et al. Osteopontin shapes immunosuppression in the metastatic niche. Cancer Res. 74, 4706-4719 (2014).

118. Welte, T. et al. Oncogenic mTOR signalling recruits myeloid-derived suppressor cells to promote tumour initiation. Nat. Cell Biol. 18 632-644 (2016).

119. Steele, C. W. et al. CXCR2 inhibition profoundly suppresses metastases and augments immunotherapy in pancreatic ductal adenocarcinoma. Cancer Cell 29, 832-845 (2016).

120. Smith, H. A. \& Kang, Y. The metastasis-promoting roles of tumorassociated immune cells. J. Mol. Med. 91, 411-429 (2013).
121. Viguier, M. et al. Foxp3 expressing CD4+CD25(high) regulatory T cells are overrepresented in human metastatic melanoma lymph nodes and inhibit the function of infiltrating T cells. J. Immunol. 173, 1444-1453 (2004).

122. Dunn, G. P., Old, L. J. \& Schreiber, R. D. The immunobiology of cancer immunosurveillance and immunoediting. Immunity 21, 137-148 (2004).

123. Giraldo, N. A. et al. The immune contexture of primary and metastatic human tumours. Curr. Opin. Immunol. 27, 8-15 (2014).

124. Eyles, J. et al. Tumor cells disseminate early, but immunosurveillance limits metastatic outgrowth, in a mouse model of melanoma. J. Clin. Invest. 120, 2030-2039 (2010).

125. Lopez-Soto, A., Gonzalez, S., Smyth, M. J. \& Galluzzi, L. Control of metastasis by NK cells. Cancer Cell 32, 135-154 (2017).

126. Schreiber, R. D., Old, L. J. \& Smyth, M. J. Cancer immunoediting: integrating immunity's roles in cancer suppression and promotion. Science 331, 1565-1570 (2011).

127. Paolino, M. et al. The E3 ligase Cbl-b and TAM receptors regulate cancer metastasis via natural killer cells. Nature 507, 508-512 (2014).

128. Bidwell, B. N. et al. Silencing of Irf7 pathways in breast cancer cells promotes bone metastasis through immune escape. Nat. Med. 18, 1224-1231 (2012).

129. Payne, K. K. et al. Tumor-reactive immune cells protect against metastatic tumor and induce immunoediting of indolent but not quiescent tumor cells. J. Leukoc. Biol. 100, 625-635 (2016).

130. Kim, R., Emi, M. \& Tanabe, K. Cancer immunoediting from immune surveillance to immune escape. Immunology 121, 1-14 (2007).

131. Rhim, A. D. et al. EMT and dissemination precede pancreatic tumor formation. Cell 148, 349-361 (2012).

132. Harper, K. L. et al. Mechanism of early dissemination and metastasis in Her2 ${ }^{+}$mammary cancer. Nature 540, 588-592 (2016).

133. Hosseini, H. et al. Early dissemination seeds metastasis in breast cancer. Nature 540, 552-558 (2016).

134. Muller, M. et al. EblacZ tumor dormancy in bone marrow and lymph nodes: active control of proliferating tumor cells by $\mathrm{CD}^{+}{ }^{+}$immune $\mathrm{T}$ cells. Cancer Res. 58, 5439-5446 (1998).

135. Aguirre-Ghiso, J. A. Models, mechanisms and clinical evidence for cancer dormancy. Nat. Rev. Cancer 7, 834-846 (2007).

136. Davies, L. C., Jenkins, S. J., Allen, J. E. \& Taylor, P. R. Tissue-resident macrophages. Nat. Immunol. 14, 986-995 (2013).

137. Fan, X. \& Rudensky, A. Y. Hallmarks of tissue-resident lymphocytes. Cell 164, 1198-1211 (2016).

138. Jimenez-Sanchez, A. et al. Heterogeneous tumor-immune microenvironments among differentially growing metastases in an ovarian cancer patient. Cell 170, 927-938 (2017).

139. Smith, H. A. \& Kang, Y. Determinants of organotropic metastasis. Annu. Rev. Cancer Biol. 1, 403-423 (2017).

140. Wu, J. B. et al. MAOA-dependent activation of Shh-IL6-RANKL signaling network promotes prostate cancer metastasis by engaging tumor-stromal cell interactions. Cancer Cell 31, 368-382 (2017).

141. Nilsson, M., Adamo, H., Bergh, A. \& Halin Bergstrom, S. Inhibition of lysyl oxidase and lysyl oxidase-like enzymes has tumour-promoting and tumour-suppressing roles in experimental prostate cancer. Sci. Rep. 6, 19608 (2016).

142. Shay, G., Lynch, C. C. \& Fingleton, B. Moving targets: Emerging roles for MMPs in cancer progression and metastasis. Matrix Biol. 44-46, 200-206 (2015).

143. Shen, S. et al. Vascular endothelial growth factor enhances cancer cell adhesion to microvascular endothelium in vivo. Exp. Physiol. 95 , 369-379 (2010).

144. Germano, G. et al. Role of macrophage targeting in the antitumor activity of trabectedin. Cancer Cell 23, 249-262 (2013).

145. Sharma, P., Hu-Lieskovan, S., Wargo, J. A. \& Ribas, A. Primary, adaptive, and acquired resistance to cancer immunotherapy. Cell 168, 707-723 (2017)

146. Schachter, J. et al. Pembrolizumab versus ipilimumab for advanced melanoma: final overall survival results of a multicentre, randomised, open-label phase 3 study (KEYNOTE-006). Lancet 390, 1853-1862 (2017).

147. Bullman, S. et al. Analysis of Fusobacterium persistence and antibiotic response in colorectal cancer. Science 358, 1443-1448 (2017).

148. Aguado, B. A., Bushnell, G. G., Rao, S. S., Jeruss, J. S. \& Shea, L. D. Engineering the pre-metastatic niche. Nat. Biomed. Eng. 1, 0077 (2017).

149. Pontes-Quero, S. et al. Dual ifgMosaic: a versatile method for multispectral and combinatorial mosaic gene-function analysis. Cell 170, 800-814 (2017).

\section{Acknowledgements}

We thank members of our laboratories for helpful discussions, and in particular, D. Liu for critical reading of the manuscript. We also apologize to the many investigators whose important studies could not be cited directly here owing to space limitations. The work was supported by a Susan G. Komen Fellowship (PDF15332075) and from Instituto de Salud Carlos III-FSE (MS17/00037) to T. C-T, and grants from the Brewster Foundation, 
the Breast Cancer Research Foundation, Department of Defense (BC123187), and the National Institutes of Health (R01CA141062) to Y.K.

\section{Competing interests}

The authors declare no competing interests.

\section{Additional information}

Reprints and permissions information is available at www.nature.com/reprints. Correspondence should be addressed to Y.K.

Publisher's note: Springer Nature remains neutral with regard to jurisdictional claims in published maps and institutional affiliations. 